\title{
Delta Opioid Receptor Agonist KNT-127 Facilitates Neuroexcitability in the Mouse Infralimbic Cortex via mTOR Pathway to Exert an Antidepressant Effect
}

\author{
Toshinori Yoshioka ${ }^{1}$, Daisuke Yamada ${ }^{1}$, Keita Iio $^{2}$, Hiroshi Nagase ${ }^{2}$, and Akiyoshi Saitoh ${ }^{1}$ \\ ${ }^{1}$ Tokyo University of Science \\ ${ }^{2}$ University of Tsukuba
}

September 25, 2021

\begin{abstract}
Background and Purpose Growing evidence demonstrates that the delta opioid receptor (DOP) is an attractive candidate for novel antidepressants with the potential to exhibit rapid action with few adverse effects. However, the underlying detailed functional mechanism remains elusive. Previously, we reported that the selective DOP agonist, KNT-127, produced robust antidepressant-like effects in the mice forced swimming test (FST). Thus, we attempted to identify the cellular mechanism underlying this effect. Experimental Approach Male ICR mice (4-6 weeks) were used in all experiments. The FST was conducted as a screening model for antidepressants. The phosphorylation level of proteins in specific brain regions was quantified using Western blotting. Glutamate/gamma-aminobutyric acid-dependent postsynaptic currents were detected using wholecell voltage-clamp recordings. Key Results The selective mTOR inhibitor, rapamycin, and the PI3K inhibitor, LY294002, blocked the antidepressant-like effects of KNT-127 in the FST. KNT-127 increased the phosphorylation level of mTOR signalrelated proteins, Akt and p70S6K, in the medial prefrontal cortex. The bilateral microinfusion of KNT-127 in the infralimbic cortex decreased immobility in the FST. The frequency of miniature excitatory postsynaptic currents in the infralimbic cortex increased and that of miniature inhibitory postsynaptic currents decreased with the perfusion of KNT-127, which was blocked by pretreatment with rapamycin. Conclusions and Implications KNT-127 displays antidepressant-like actions through the direct facilitation of neuronal excitability in the mice infralimbic cortex, which is implicated in the PI3K-Akt-mTOR-p70S6K signaling pathway. These results could indicate the first steps in elucidating the complete mechanical functions of DOPs as a potential candidate for novel antidepressants.
\end{abstract}

Delta Opioid Receptor Agonist KNT-127 Facilitates Neuroexcitability in the Mouse Infralimbic Cortex via mTOR Pathway to Exert an Antidepressant Effect

Short running title : Functional mechanism of the antidepressant-like effect of KNT-127

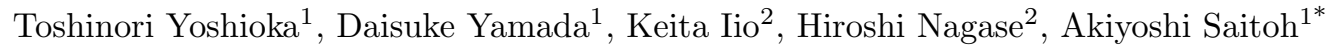

${ }^{1}$ Laboratory of Pharmacology, Faculty of Pharmaceutical Science, Tokyo University of Science, Noda, Japan

${ }^{2}$ International Institute for Integrative Sleep Medicine, University of Tsukuba, Ibaraki, Japan

*Corresponding author : Akiyoshi Saitoh

Address: 2641 Yamazaki, Noda-shi, Chiba Prefecture 278-8510, Japan

Phone: +81-4-7121-3610

Email:akiyoshi_saitoh@rs.tus.ac.jp 
ORCID: https://orcid.org/0000-0001-8617-2441

\section{Word count}

Abstract: 248 words

Introduction: 475 words

Methods: 1708 words

Results: 772 words

Discussion and Conclusions: 954 words

\section{Data availability}

The data that support the findings of this study are available from the corresponding author upon reasonable request.

\section{Acknowledgments}

This research was supported by funding received from the Cyclic Innovation for Clinical Empowerment as part of the Japan Agency for Medical Research and Development (AMED) under Grant Number 17pc0101018h0001.

\section{Author contribution}

TY performed most of the operations of the study. DY provided technical and intellectual advice. KI synthesized the compound. HN and AS supervised the project. All authors contributed to the article and approved the submitted version.

\section{Conflict of interest disclosure}

The authors declare no conflicts of interest.

\section{Declaration of transparency and scientific rigour}

This Declaration acknowledges that this paper adheres to the principles for transparent reporting and scientific rigour of preclinical research as stated in the BJP guidelines for Design and Analysis, Immunoblotting and Immunochemistry, and Animal Experimentation, and as recommended by funding agencies, publishers and other organisations engaged with supporting research.

- What is already known

- A selective delta opioid receptor agonist, KNT-127, exerts antidepressant-like effects in rodent studies.

- What this study adds

- KNT-127 produces antidepressant-like effects in the mouse infralimbic cortex involved in the PI3KmTOR signaling.

- KNT-127 accelerates glutamatergic neurotransmitter response by suppressing the GABAergic system in mouse infralimbic cortex.

- Clinical significance

- New findings on delta opioid receptors mechanism indicate a potential candidate for novel antidepressants.

\section{Abstract}

\section{Background and Purpose}

Growing evidence demonstrates that the delta opioid receptor (DOP) is an attractive candidate for novel antidepressants with the potential to exhibit rapid action with few adverse effects. However, the underlying detailed functional mechanism remains elusive. Previously, we reported that the selective DOP agonist, 
KNT-127, produced robust antidepressant-like effects in the mice forced swimming test (FST). Thus, we attempted to identify the cellular mechanism underlying this effect.

\section{Experimental Approach}

Male ICR mice (4-6 weeks) were used in all experiments. The FST was conducted as a screening model for antidepressants. The phosphorylation level of proteins in specific brain regions was quantified using Western blotting. Glutamate/gamma-aminobutyric acid-dependent postsynaptic currents were detected using wholecell voltage-clamp recordings.

\section{Key Results}

The selective mTOR inhibitor, rapamycin, and the PI3K inhibitor, LY294002, blocked the antidepressantlike effects of KNT-127 in the FST. KNT-127 increased the phosphorylation level of mTOR signal-related proteins, Akt and p70S6K, in the medial prefrontal cortex. The bilateral microinfusion of KNT-127 in the infralimbic cortex decreased immobility in the FST. The frequency of miniature excitatory postsynaptic currents in the infralimbic cortex increased and that of miniature inhibitory postsynaptic currents decreased with the perfusion of KNT-127, which was blocked by pretreatment with rapamycin.

\section{Conclusions and Implications}

KNT-127 displays antidepressant-like actions through the direct facilitation of neuronal excitability in the mice infralimbic cortex, which is implicated in the PI3K-Akt-mTOR-p70S6K signaling pathway.

These results could indicate the first steps in elucidating the complete mechanical functions of DOPs as a potential candidate for novel antidepressants.

Keywords : antidepressants, delta opioid receptor, GABA, glutamate, mTOR, prefrontal cortex

\section{Introduction}

It has become widely accepted that delta opioid receptors (DOPs) play key roles in mood disorders (1). For example, mice deficient in DOPs exhibit depressive and anxiety-like behaviors (2), and the inhibition of enkephalinase, which degrades an endogenous DOP ligand, enkephalin, produces antidepressant-like behavior in rats (3). Moreover, we previously reported that the DOP agonist demonstrated antidepressant and anxiolytic-like effects in rodents (4). In addition, we reported that the selective DOP agonist, KNT-127 (delta: $\mathrm{K}_{\mathrm{i}}=0.16 \mathrm{nM}$, kappa: $\mathrm{K}_{\mathrm{i}}=153 \mathrm{nM}, \mathrm{mu}: \mathrm{K}_{\mathrm{i}}=21.3 \mathrm{nM} ; 5$ ), shows antidepressant-like effects in the forced swimming test (FST) and the olfactory bulbectomized model (6,7). Furthermore, in rodents, KNT127 has anxiolytic-like effects in the elevated plus maze test, open field test, and light-dark test (8-10). Notably, to the best of our knowledge, KNT-127 has no adverse effects such as memory impairment, motor coordination deficits, ethanol interactions, convulsions, or digestive symptoms $(6,8,11)$. Thus, we propose the possibility that DOP is a novel target as a psychotropic drug for emotional disorders. Nevertheless, detailed functional mechanisms of those effects remain unclear.

Recently, the mechanistic (or mammalian) target of rapamycin (mTOR) has been suggested to play an important role in the molecular mechanisms underlying antidepressant-like effects in the rodents' medial prefrontal cortex (mPFC) and hippocampus (12-14). The activation of the mTOR signaling pathway that spurs synaptogenesis in the mPFC contributes to the rapid antidepressant-like effect of ketamine (15). The TrkB-dependent mTOR signal activation, which results in the acceleration of neural progenitor cell differentiation in the hippocampus, is involved in the sustained antidepressant-like effect of ketamine (16). Moreover, chronic fluoxetine treatment has relieved chronic, unpredictable mild stress-induced behavioral deficits through a signal transduction by mTOR in the hippocampus (17).

Multiple studies have reported that the change in glutamatergic neurotransmission is highly associated with the pathophysiological condition of patients with mood disorders (18). In 1990, it was demonstrated that Nmethyl-D-aspartate (NMDA) receptor antagonists exhibit antidepressant-like actions in mice (19). The past two decades have revealed that ketamine, an NMDA receptor antagonist, showed rapid antidepressant-like 
effects in preclinical (15) and clinical studies (20). Furthermore, abnormalities in the monoaminergic system, often observed in depressive and anxiety states, also alter the glutamatergic system; thus, the "monoamine hypothesis" of depression is being transposed into the "glutamate hypothesis" (21). In recent clinical studies, magnetic resonance spectroscopy and positron emission tomography clarified that glutamate and gammaaminobutyric acid (GABA) concentrations altered in patients with major depressive disorder (MDD), which causes dysfunction in excitatory and/or inhibitory neurotransmitter regulation (22).

In the present study, we attempted to elucidate the cellular mechanism of the antidepressant-like effect of the selective DOP agonist, KNT-127. Accordingly, we examined 1) whether mTOR signal transduction is involved in its effect, 2) the specific brain region(s) in which its effect is exerted, and 3) the relation between glutamatergic and/or GABAergic neuronal responses and its effect.

\section{Methods}

\subsection{Animals}

The animal species, strain, and sex were selected as in our previous study (6). Male ICR mice weighing 20-30 g and aged 4-5 weeks were obtained from CLEA Japan. They were divided into equal groups, as shown in the figures and housed at 4-6 per cage $(225 \times 338 \times 140 \mathrm{~mm})$ for stratified randomization and acclimatized to the breeding room for at least five days before behavioral tests were performed. Mice were housed under controlled air temperature and pressure and kept in $12 \mathrm{~h}$ light/dark cycles (lights on from 08:00 to 20:00) with ad libitumfood and water. All behavioral tests were performed during the light cycle. All animal experiments were conducted in accordance with the guidelines formulated by the animal welfare committee of the Tokyo University of Science (Approval Nos. Y19032 and Y20020) and in compliance with the ARRIVE guidelines (23) and the recommendations of the British Journal of Pharmacology (24).

\subsection{Drug administration}

KNT-127 and naltrindole (Merck KGaA, Darmstadt, Germany) were dissolved in saline; rapamycin (LC Laboratories, Woburn, MA), LY294002 (FUJIFILM Wako Pure Chemical, Osaka, Japan), and U0126 (FUJIFILM Wako Pure Chemical) were dissolved in dimethyl sulfoxide, followed by dilution with saline for the final concentration. For a systemic administration, KNT-127 (10 $\left.\mathrm{mg} \mathrm{kg}^{-1}\right)$ was injected subcutaneously (s.c.) 30 min before the behavioral test or sacrifice, unless otherwise stated. For intracerebroventricular (i.c.v.) injection, under isoflurane inhalation anesthesia, a 27-gauge injection needle with stopper (TOP Corporation, Tokyo, Japan) was inserted into the mice using a microsyringe (Hamilton Company, Reno, NV), perpendicularly into +-1.0 $\mathrm{mm}$ (medial/lateral, ML) and -3.0 $\mathrm{mm}$ (dorsal/ventral, DV) from the bregma (25), and $5 \mu \mathrm{L}$ drug solution was gently infused. Each inhibitor was injected 60 min before the behavioral test or sacrifice. For local infusion, mice were anesthetized with the intraperitoneal injection of a mixture of medetomidine $\left(0.75 \mathrm{mg} \mathrm{kg}^{-1}\right)$, midazolam $\left(4 \mathrm{mg} \mathrm{kg}^{-1}\right)$, and butorphanol $\left(5 \mathrm{mg} \mathrm{kg}^{-1}\right)$, checked for the absence of the tail-pinch reflux, restrained onto a stereotaxic apparatus, administered ophthalmic ointment, and implanted with guide cannula(s) into target regions bilaterally seven days before the test for a recovery period. After the surgery, the mice were given atipamezole $\left(0.75 \mathrm{mg} \mathrm{kg}^{-1}\right)$ and placed on a warm mat for at least $1 \mathrm{~h}$. Then $15 \mathrm{~min}$ before the behavioral test, the mice were infused with $0.2 \mu \mathrm{L} /$ side of KNT-127 ( $1 \mathrm{mM})$ with the microsyringe pump at the speed of $0.2 \mu \mathrm{L} \mathrm{min}{ }^{-1}$, and the injection cannula was allowed to remain for one min after the injection. For prelimbic cortex (PL-PFC) infusion, a 26-gauge dual guide cannula (0.8 $\mathrm{mm}$ interval; P1 Technologies, Roanoke, VA) was placed at $+1.9 \mathrm{~mm}$ (anterior/posterior, AP), $\pm 0.4 \mathrm{~mm}$ (ML), and $-2.4 \mathrm{~mm}$ (DV) from the bregma (25). For infralimbic cortex (IL-PFC) infusion, 25-gauge guide cannulas (Eicom, Kyoto, Japan) were placed at $+1.7 \mathrm{~mm}$ (AP), +-0.4 $\mathrm{mm}$ (ML), and $-3.0 \mathrm{~mm}$ (DV) from the bregma (25) and angled at 30deg to the sagittal plane to avoid damaging the PL-PFC.

\subsection{FST}

The FST procedure was performed as previously reported with minor modifications (6). A 5-L glass cylinder (diameter, $175 \mathrm{~mm}$; height, $265 \mathrm{~mm}$ ) filled with 25 +- 1 degC water to $3 \mathrm{~L}$ (depth, $131 \mathrm{~mm}$ ) was used as a swimming apparatus. On the first day, mice were individually immersed in water for $15 \mathrm{~min}$ as a training 
session. Subsequently, $24 \mathrm{~h}$ later, each animal was put into the apparatus for $10 \mathrm{~min}$ as a test session, and dominant behaviors were counted manually at $5 \mathrm{~s}$ intervals during the final $6 \mathrm{~min}$. Immobility was defined as mice stopping all active behavior and floating with minimal movement. After each test, the i.c.v. injection or cannula implantation sites were verified, and the subject where the position was inappropriate was discarded from the data.

\subsection{Locomotor activity measurement}

Mice were placed in a fresh acrylic cage $(310 \times 205 \times 125 \mathrm{~mm})$ and acclimatized for $2 \mathrm{~h}$. Following drug administration, locomotion was counted with an automatic tracking system (Muromachi Kikai, Tokyo, Japan) every 5 min for $40 \mathrm{~min}$.

\subsection{Western blotting}

Western blotting was conducted in conformity with the British Journal of Pharmacology guidelines on immunoblotting (26) with the exception that statistical comparisons of protein bands were assessed through different blots due to the sample size (27). Immediately after the dislocation of the cervical vertebra, the brains were extracted. Subsequently, the mPFC, amygdala, and hippocampus were dissected from the brains and rapidly frozen in liquid nitrogen and stored at $-80 \mathrm{degC}$ until use. Samples were homogenized in a cell lysis buffer (1\% Triton X-100, 0.1\% SDS, $50 \mathrm{mM}$ Tris-HCl, $150 \mathrm{mM} \mathrm{NaCl}, 2 \mathrm{mM} \mathrm{K}{ }_{2}$ EDTA, phosphatase inhibitors, and protease inhibitors), followed by centrifuging at 2,500 $\mathrm{x}$ for $15 \mathrm{~min}$ at $4 \mathrm{degC}$. A supernatant was used for immunoblotting, and the protein concentration was determined with the BCA protein assay kit (Thermo Fisher Scientific, Waltham, MA). For immunoblotting, $6 \mu \mathrm{g}$ of proteins and $4 \mu \mathrm{L}$ of molecular weight marker (NIPPON genetics, Tokyo, Japan) were loaded into 7.5\%-10\% SDS-PAGE gel for electrophoresis and subsequently transferred to PVDF membranes (Bio-Rad, Hercules, CA). Membranes were blocked by $5 \%$ skim milk (MORINAGA, Tokyo, Japan) or bovine serum albumin (FUJIFILM Wako Pure Chemical) in TBS-T (50 mM Tris, $138 \mathrm{mM} \mathrm{NaCl}, 2.7 \mathrm{mM} \mathrm{KCl}, 0.1 \%$ Tween-20, $\mathrm{pH} 7.4$ ) for $1 \mathrm{~h}$ at room temperature and incubated for $1 \mathrm{~h}$ at room temperature with the following primary antibodies in the blocking solution (used only once): p-Akt (rabbit polyclonal; 1:1000; Cat\# 9271, RRID:AB_329825, Cell Signaling Technology, Danvers, MA), total Akt (rabbit polyclonal; 1:1000; Cat\# 9272, RRID:AB_329827, Cell Signaling Technology), p-ERK (rabbit polyclonal; 1:1000; Cat\# 9101, RRID:AB_331646, Cell Signaling Technology), total ERK (rabbit polyclonal; 1:1000; Cat\# 9102, RRID:AB_330744, Cell Signaling Technology), p-p70S6K (rabbit monoclonal; 1:1000; Cat\# 9234, RRID:AB_2269803, Cell Signaling Technology), and total p70S6K (rabbit monoclonal; 1:1000; Cat\# 2708, RRID:AB_390722, Cell Signaling Technology). After a rinse with TBS-T, the membrane was incubated with horseradish peroxidase-conjugated anti-rabbit secondary antibodies in the blocking solution (used only once; goat polyclonal; 1:5000; Cat\# ab6721, RRID:AB_955447, abcam, Cambridge, UK) for $1 \mathrm{~h}$ at room temperature. Signals were detected using an enhanced chemiluminescence system (Merck KGaA) and captured with LAS-4000 (FUJIFILM, Tokyo, Japan). Detections of phosphorylated and total proteins were performed from different blots to avoid a membrane-stripping process. Protein bands were quantified densitometrically using imageJ software (NIH, Bethesda, MD).

\subsection{Acute slice preparation and whole-cell patch-clamp recordings}

The procedure for electrophysiology was performed as previously reported with minor modifications $(28,29)$. The animals were deeply anesthetized with isoflurane, and their brains were extracted and placed in cold artificial cerebrospinal fluid (aCSF: $124 \mathrm{mM} \mathrm{NaCl}, 3 \mathrm{mM} \mathrm{KCl}, 1.2 \mathrm{mM} \mathrm{KH}_{2} \mathrm{PO}_{4}, 26 \mathrm{mM} \mathrm{NaHCO}, 2 \mathrm{mM}$ $\mathrm{CaCl}_{2}, 1.3 \mathrm{mM} \mathrm{MgSO}_{4}$, and $10 \mathrm{mM}$ d-glucose) and continuously bubbled with $95 \% \mathrm{O}_{2} / 5 \% \mathrm{CO}_{2}$ for 2 min. Subsequently, a 300- $\mu \mathrm{m}$ thickness of coronal sections containing IL-PFC was obtained on a vibrating blade tissue slicer (LinearSlicer PRO7N, DOSAKA EM, Kyoto, Japan) and immersed in aCSF at room temperature for at least $30 \mathrm{~min}$ as a recovery period. A single slice was placed in a submersion chamber and perfused aCSF at 2-4 $\mathrm{mL} \mathrm{min}^{-1}$ (gravity flow) warmed at $32{ }^{\circ} \mathrm{C}$. Pyramidal neurons in layer II/III or layer $\mathrm{V}$ of the IL-PFC were identified with microscopy ( $\times 400$ total; IR/DIC; BX51WI, Olympus, Tokyo, Japan). The output signal was amplified and filtered at $2 \mathrm{kHz}$ using an AXOPATCH 200B patch-clamp amplifier (Molecular Devices, San Jose, CA). Data were digitized at $5 \mathrm{kHz}$ and acquired using Clampex software 
version 9.2 (Molecular Devices). Series resistance, which was frequently monitored during recording, ranged from 11 to $20 \mathrm{M} \Omega$. cells were discarded from the analysis if the values changed by more than $20 \%$ or exceeded $25 \mathrm{M} \Omega$.

\subsection{Analysis of miniature excitatory postsynaptic currents (mEPSCs)}

Patch electrodes (resistance 4-6 M $\Omega$ ) were filled with a solution containing the following: $132 \mathrm{mM} \mathrm{K}$ gluconate, $3 \mathrm{mM} \mathrm{KCl}, 10 \mathrm{mM}$ HEPES, $0.5 \mathrm{mM}$ EGTA, $1 \mathrm{mM} \mathrm{MgCl} 2,12 \mathrm{mM}$ sodium phosphocreatine, $3 \mathrm{mM}$ ATP magnesium salt, and $0.5 \mathrm{mM} \mathrm{GTP}$, adjusted to $\mathrm{pH} 7.4$ with $\mathrm{KOH}$. Postsynaptic currents were studied in voltage-clamp mode with clamped estimated resting potential of -65 $\mathrm{mV}$. Immediately after establishing the whole-cell configuration, a series of depolarizing rectangular current steps $(750 \mathrm{~ms} ; 20 \mathrm{pA}$ increments; interpulse interval $3 \mathrm{~s}$ ) and spontaneous postsynaptic currents were verified. Subsequently, tetrodotoxin (1 $\mu \mathrm{M})$ was perfused for 5 min to block voltage-gated sodium channels, and miniature postsynaptic currents were recorded for $1 \mathrm{~min}$. KNT-127 $(10 \mu \mathrm{M})$ was then perfused for $15 \mathrm{~min}$, and the currents were again recorded for $1 \mathrm{~min}$. Traces were analyzed using software (Mini Analysis Program, v.6.0.7, Synaptosoft, Decatur, GA). The threshold amplitude for current detection was $7.5 \mathrm{pA}$, and the times required for a $10 \%^{-}$ $90 \%$ rise and a $37 \%$ decay in response were taken as the rise and decay times, respectively. Each value was compared before and after the application of KNT-127.

\subsection{Analysis of miniature inhibitory postsynaptic currents (mIPSCs)}

Similar to the mEPSC recordings, mIPSC recordings were performed (see Section 2.7). The points of difference are as follows: the internal solution of the patch electrodes contained $105 \mathrm{mM} \mathrm{K}$-gluconate, 30 $\mathrm{mM} \mathrm{KCl}, 10 \mathrm{mM}$ HEPES, $0.5 \mathrm{mM}$ EGTA, $1 \mathrm{mM} \mathrm{MgCl}_{2}, 12 \mathrm{mM}$ sodium phosphocreatine, $3 \mathrm{mM}$ ATP magnesium salt, and $0.5 \mathrm{mM}$ GTP; the membrane potential was clamped at $-70 \mathrm{mV}$; and CNQX $(20 \mu \mathrm{M})$ and $d$-AP5 $(50 \mu \mathrm{M})$, in addition to tetrodotoxin, were perfused to block AMPA and NMDA receptors.

\subsection{Materials}

All reagents used in the drug administration, Western blotting, and electrophysiology were purchased from FUJIFILM Wako Pure Chemical or Merck KGaA.

\subsection{Data and statistical analysis}

Data and statistical analysis complied with the recommendations of theBritish Journal of Pharmacology for experimental design and analysis in pharmacology (27), with the exception that data acquisition and analysis were not blinded due to technical constraints. All sample sizes were determined by reference to our previous reports $(6,28,30)$. All data are presented as means \pm standard error of the mean (S.E.M.). The analysis was performed using GraphPad Prism7 (GraphPad Software, San Diego, CA). Two groups' data were analyzed using the Student'st -test or the paired sample $t$-test; the remaining groups were analyzed using one-way or two-way factorial ANOVA, followed bypost-hoc. Dunnett's test compared the test group to the control group. Statistical significance was defined as ${ }^{*} p<0.01$.

\section{Results}

\subsection{KNT-127 exerts antidepressant-like effects through DOP.}

First, we performed the FST to assess the proper dose and onset time of KNT-127 in the present laboratory circumstance. A single injection of KNT-127 (10 mg kg-1, s.c.) 30 min before the test significantly decreased immobility counts (Figure 1A) but did not affect locomotor activity counts compared to the vehicle-treated group (Figures 1B). Moreover, the effect of KNT-127 in the FST was blocked by pretreatment with the DOP inhibitor, naltrindole (10 nmol, i.c.v.), (two-way ANOVA; main effect of agonist: $F_{(1,33)}=18.71, p<0.01$; main effect of inhibitor: $F_{(1,33)}=27.66, p<0.01$; interaction effects: $F_{(1,33)}=10.84, p<0.01$; Figure $1 \mathrm{C})$. Consistent with an earlier report (6), these results indicate that KNT-127 exerts an antidepressant-like action through DOP.

\subsection{KNT-127 activates mTOR signaling pathway in the mPFC and amygdala .}


Second, to investigate whether the antidepressant-like effect of KNT-127 requires an mTOR dependent pathway, we performed the preinfusion of the selective mTOR inhibitor, rapamycin (0.2 nmol, i.c.v.), 30 min before the administration of KNT-127 (10 mg kg-1 , s.c.) in the FST. Rapamycin completely reversed immobility counts attenuated by KNT-127 (two-way ANOVA; main effect of agonist: $F_{(1,31)}=7.593, p$ $<0.01$; main effect of inhibitor: $F_{(1,31)}=2.716$, nonsignificant; interaction effects: $F_{(1,31)}=4.080$, nonsignificant; Figure 1D), suggesting that mTOR signal transduction was involved in the antidepressantlike effect of KNT-127. Subsequently, we analyzed the activation of mTOR signaling related proteins in specific brain regions, namely, the mPFC, amygdala, and hippocampus, which are commonly known to be closely implicated regions in mood disorders, using Western blotting. KNT-127 increased the phosphorylation level of one upstream protein of mTOR, Akt, in the mPFC (two-way ANOVA; main effect of agonist: $F$ $(1,16)=43.71, p<0.01$; main effect of inhibitor: $F_{(1,16)}=0.5829$, nonsignificant; interaction effects: $F_{(1,16)}$ $=0.07908$, nonsignificant; Figure $2 \mathrm{~A}$ and, on the other hand, ERK in the amygdala (two-way ANOVA; main effect of agonist: $F_{(1,16)}=153.6, p<0.01$; main effect of inhibitor: $F_{(1,16)}=0.01744$, nonsignificant; interaction effects: $F_{(1,16)}=0.01036$, nonsignificant; Figure 2B). Simultaneously, a downstream protein of mTOR, p70S6K, increased the phosphorylation level by the administration of KNT-127 in the mPFC and the amygdala, which was inhibited by rapamycin as expected (Figure 2C). Thus, the antidepressant-like effects of KNT-127 might pass through Akt-mTOR-p70S6K in the mPFC and/or ERK-mTOR-p70S6K in the amygdala.

\subsection{Antidepressant-like effects of KNT-127 are inhibited by a PI3K-Akt inhibitor.}

To examine if those pathways are involved in the antidepressant-like effect of KNT-127, we used the inhibitors of the upstream proteins Akt or ERK. As a result, pretreatment with the PI3K inhibitor, LY294002 (20 nmol, i.c.v.), definitely abolished the antidepressant-like effect of KNT-127 (10 mg kg-1, s.c.) in the FST (two-way ANOVA; main effect of agonist: $F_{(1,33)}=8.147, p<0.01$; main effect of inhibitor: $F_{(1,33)}$ $=3.133$, nonsignificant; interaction effects: $F_{(1,33)}=5.078, \mathrm{p}=0.0310$; Figure 3A), whereas an MEK inhibitor, U0126 (20 nmol, i.c.v.), had no effect (two-way ANOVA; main effect of agonist: $F_{(1,34)}=40.03$, $p<0.01$; main effect of inhibitor: $F_{(1,34)}=0.05808$, nonsignificant; interaction effects: $F_{(1,34)}=0.01046$, nonsignificant; Figure 3B). Therefore, the effects of KNT-127 were evoked through the PI3K-Akt pathway.

\subsection{KNT-127 locally produces antidepressant-like behavior effects in the IL-PFC.}

Following the aforementioned results, we tested whether KNT-127 directly displays antidepressant-like action in the mPFC. A microinfusion of KNT-127 $(1 \mathrm{mM}, 0.2 \mu \mathrm{L} /$ side $)$ into the PL-PFC did not significantly diminish immobility counts (Figure 4A). By contrast, KNT-127 attenuated counts in the IL-PFC (Figure $4 \mathrm{~B})$.

\subsection{KNT-127 accelerates glutamate release in the IL-PFC with the involvement of the mTOR} signaling pathway.

To determine the physiological mechanism of KNT-127 to exert antidepressant-like action, we then evaluated influences of KNT-127 $(10 \mu \mathrm{M})$ on the mEPSCs of pyramidal neurons in the IL-PFC. As shown in Figure 5, the mean frequency, but not the amplitude, rise time, or decay time, of the mEPSCs significantly increased with the perfusion of KNT-127 (Figures 5A-5E), suggesting that KNT-127 promoted glutamate release in the presynaptic site. Furthermore, the pretreatment of mice with rapamycin ( $0.2 \mathrm{nmol}$, i.c.v.) reversed the enhanced frequency (Figures $5 \mathrm{~F}-5 \mathrm{~J}$ ).

\subsection{KNT-127 suppresses GABAergic synapse currents in the IL-PFC.}

Finally, we investigated whether the KNT-127-induced excitability of IL-PFC pyramidal neurons was implicated in the GABAergic system. The mean frequency of the mIPSCs significantly decreased with the perfusion of KNT-127 (Figures 6A-6E), which is also inhibited by rapamycin (Figures 6F-6J). These results suggest that KNT-127 suppressed GABA release, leading to glutamatergic neuronal activation.

\section{Discussion and Conclusions}


We have proposed that the DOP has substantial potential as a novel and valuable drug candidate for mood disorders such as depression without producing the adverse effects related to benzodiazepine anxiolytics and/or monoaminergic antidepressants. In the present study, we attempted to clarify the functional mechanisms of antidepressant-like actions of the selective DOP agonist, KNT-127. Consequently, the systemic administration of KNT-127 attenuated immobility counts in the mice FST, inhibited by pretreatment with rapamycin, indicating that KNT-127 produced antidepressant-like effects via the activation of the mTOR signaling pathway. In addition, KNT-127 elevated the phosphorylation of Akt and p70S6K in the mPFC; furthermore, a PI3K inhibitor, LY294002, blocked the antidepressant-like effects of KNT-127. Moreover, the local infusion of KNT-127 into the IL-PFC, but not the PL-PFC, exhibited robust antidepressant-like action. Finally, we demonstrated that KNT-127 accelerated glutamatergic neurotransmitter response by suppressing the GABAergic system in the IL-PFC. Thus, we proposed the cellular mechanism of KNT-127's antidepressant-like effects in that the drug produced antidepressant-like effects via DOP owing to the presynaptic promotion of glutamate release in the IL-PFC, which was implicated in the PI3K-Akt-mTOR-p70S6K signal transduction pathway.

The IL-PFC in rodents is regarded as equivalent to Brodmann Area 25 because of its functions in mood regulation and projections to other brain regions $(31,32)$. Clinical studies have reported that Brodmann Area 25 correlates with negative mood, such as a depressive state (33), and with MDD patients' treatment resistance (34). Furthermore, in MDD patients, the area was reduced in volume and hypoactivated (35). In addition, the novel antidepressant ketamine has recently been reported to produce rapid and sustained antidepressant- and anxiolytic-like actions, accompanied by the synaptic response of pyramidal neurons in rats IL-PFC (36). A single bilateral infusion of $R$-ketamine into the IL-PFC also shows antidepressant effects in the learned helplessness model rats (37). In this study, we also revealed that DOP in the IL-PFC, but not the PL-PFC, play an important role in expression of antidepressant-like effects.

Numerous studies have revealed that depression's pathophysiology is associated with an imbalance of excitatory and inhibitory neurotransmission in the mPFC (38). According to our results, KNT-127 increases the frequency, but not the amplitude, of mEPSCs and decreases that of mIPSCs of layer II/III and V pyramidal neurons of the IL-PFC, suggesting that KNT-127 accelerates glutamate release from the presynaptic terminals of excitatory neurons by inhibiting the GABAergic system. Intriguingly, our recent report indicated that KNT-127 attenuated mEPSC frequency in the PL-PFC (39), indicating that the response to KNT-127 is completely opposite in the PL-PFC and the IL-PFC; namely, KNT-127 could play a bidirectional role in either excitatory or inhibitory neurotransmission in the PFC. Thus, DOP might modulate the glutamate/GABA system in each specific brain region.

mTOR is well known as a serine/threonine kinase and represents a component of at least two types of complexes: mTOR complex 1 (mTORC1) and mTOR complex 2 (mTORC2). mTORC1 is associated with the regulation of cellular processes, including DNA transcription, protein synthesis, cell proliferation, and cell growth (40); mTORC2 primarily contributes to cell survival and the organization of the cytoskeleton (41). In a cultured glutamatergic hippocampal neurons study, McCabe et al. (42) indicated that the genetic inactivation of either mTORC1 or mTORC2 reduced evoked EPSCs and that both complexes have a differential role in the presynaptic release of glutamate and postsynaptic reactivity, respectively. Further, presynaptic protein synthesis through the mTORC1 pathway is required for the long-term depression of the inhibitory neurotransmitter in the GABAergic neuron axon $(43,44)$. Moreover, Akt is an upstream protein of mTORC1 involved in two common pathways: PI3K-Akt-mTORC1 and mTORC2-Akt-mTORC1 (45). Given that, in our data, the selective mTOR inhibitor, rapamycin, and the PI3K inhibitor, LY294002, completely blocked antidepressant-like actions of KNT-127. Thus, in addition to the findings that rapamycin acutely and selectively inhibits mTORC1 $(44,46)$, KNT-127 might activate the PI3K-Akt-mTORC1 pathway.

For the aforementioned reason, we proposed the hypothesis that, as a functional mechanism of antidepressantlike effects, the activation of DOPs in the IL-PFC suppressed GABA release via the PI3K-Akt-mTORC1 signaling pathway, which facilitates glutamate release owing to exerted antidepressant-like effects (Figure 7). However, our experiments are not sufficient enough to conclude whether DOP-induced mTORC1 activation 
contributes presynaptically or postsynaptically. If the $\operatorname{mTOR}(\mathrm{C} 1)$ pathway could be specifically inactivated in glutamatergic or GABAergic neurons, the limitation could be addressed.

Notably, although KNT-127 promotes the phosphorylation of ERK and p70S6K in the amygdala, pretreatment with the MEK inhibitor, U0126, did not affect decreased immobility counts of KNT-127 in the mice FST, suggesting that the MEK-ERK pathway is not involved in the drug's antidepressant-like effects. The amygdala is known to be closely correlated with anxiety and emotional learning, such as fear memory $(47,48)$. In a previous study, we found that KNT-127 showed robust anxiolytic-like effects in the rat elevated plus maze test (8) and facilitated the extinction learning of contextual fear memory in the mice fear conditioning test (30). In addition, we reported that the perfusion of KNT-127 in the PL-PFC reduced veratrine-induced anxiety-like behaviors by suppressing excessive extracellular glutamate concentration in mice in vivo brain microdialysis experiments (10). Because the amygdala-PL-PFC circuit modulates anxiety-like behavior (49), we hypothesize that the activation of the ERK-mTOR-p70S6K pathway in the amygdala contributes to the anxiolytic-like effect of KNT-127. Recently, Ko et al. (50) demonstrated that ERK activation in the amygdala is critical for DOP-induced anxiolytic-like effects. Such enthralling findings strongly support the aforementioned supposition, implying that more research is required.

In conclusion, the present investigation proved that the selective DOP agonist, KNT-127, displays antidepressant-like actions through a direct acceleration of glutamate release in the IL-PFC of mice, which is involved in the PI3K-Akt-mTOR-p70S6K signal transduction pathway. These results might constitute the first step in elucidating the complete mechanical functions of DOPs as a candidate for new antidepressants.

\section{References}

1. Lutz P.E., Kieffer B.L. (2013). Opioid receptors: distinct roles in mood disorders. Trends Neurosci, 36(3): 195-206. DOI: 10.1016/j.tins.2012.11.002.

2. Filliol D., Ghozland S., Chluba J., Martin M., Matthes H.W., Simonin F., Befort K., Gavériaux-Ruff C., Dierich A., LeMeur M., Valverde O., Maldonado R., Kieffer B.L. (2000). Mice deficient for delta- and mu-opioid receptors exhibit opposing alterations of emotional responses. Nat Genet, 25(2): 195-200. DOI: $10.1038 / 76061$.

3. Jutkiewicz E.M., Torregrossa M.M., Sobczyk-Kojiro K., Mosberg H.I., Folk J.E., Rice K.C., Watson S.J., Woods J.H. (2006). Behavioral and neurobiological effects of the enkephalinase inhibitor RB101 relative to its antidepressant effects. Eur J Pharmacol , 531(1-3):151-159. DOI: 10.1016/j.ejphar.2005.12.002.

4. Saitoh A., Kimura Y., Suzuki T., Kawai K., Nagase H., Kamei J. (2004). Potential anxiolytic and antidepressant-like activities of SNC80, a selective delta-opioid agonist, in behavioral models in rodents. J Pharmacol Sci , 95(3): 374-380. DOI: 10.1254/jphs.fpj04014x.

5. Nagase H., Nemoto T., Matsubara A., Saito M., Yamamoto N., Osa Y., Hirayama S., Nakajima M., Nakao K., Mochizuki H., Fujii H. (2010). Design and synthesis of KNT-127, a $\delta$-opioid receptor agonist effective by systemic administration. Bioorg Med Chem Lett , 20(21): 6302-6305. DOI: 10.1016/j.bmcl.2010.08.083.

6. Saitoh A., Sugiyama A., Nemoto T., Fujii H., Wada K., Oka J., Nagase H., Yamada M. (2011). The novel $\delta$ opioid receptor agonist KNT-127 produces antidepressant-like and antinociceptive effects in mice without producing convulsions. Behav Brain Res , 223(2): 271-279. DOI: 10.1016/j.bbr.2011.04.041.

7. Gotoh L., Saitoh A., Yamada M., Fujii H., Nagase H., Yamada M. (2017). Effects of repeated treatment with a delta opioid receptor agonist KNT-127 on hyperemotionality in olfactory-bulbectomized rats.Behav Brain Res , 323: 11-14. DOI: 10.1016/j.bbr.2016.11.008.

8. Saitoh A., Sugiyama A., Yamada M., Inagaki M., Oka J., Nagase H., Yamada M. (2013). The novel $\delta$ opioid receptor agonist KNT-127 produces distinct anxiolytic-like effects in rats without producing the adverse effects associated with benzodiazepines. Neuropharmacology , 67: 485-493. DOI: 10.1016/j.neuropharm.2012.11.025. 
9. Sugiyama A., Nagase H., Oka J., Yamada M., Saitoh A. (2014). DOR(2)-selective but not DOR(1)-selective antagonist abolishes anxiolytic-like effects of the $\delta$ opioid receptor agonist KNT127.Neuropharmacology , 79: 314-320. DOI: 10.1016/j.neuropharm.2013.11.021.

10. Saitoh A., Suzuki S., Soda A., Ohashi M., Yamada M., Oka J., Nagase H., Yamada M. (2018). The delta opioid receptor agonist KNT-127 in the prelimbic medial prefrontal cortex attenuates veratrine-induced anxiety-like behaviors in mice. Behav Brain Res , 336: 77-84. DOI: 10.1016/j.bbr.2017.08.041.

11. Nagase H., Saitoh A. (2020): Research and development of $\chi$ opioid receptor agonists and $\delta$ opioid receptor agonists. Pharmacol Ther, 205: 107427. DOI: 10.1016/j.pharmthera.2019.107427.

12. Jernigan C.S., Goswami D.B., Austin M.C., Iyo A.H., Chandran A., Stockmeier C.A., Karolewicz B. (2011). The mTOR signaling pathway in the prefrontal cortex is compromised in major depressive disorder.Prog Neuropsychopharmacol Biol Psychiatry , 35(7): 1774-1779. DOI: 10.1016/j.pnpbp.2011.05.010.

13. Xu D., Wang C., Zhu X., Zhao W., Jiang B., Cui S., Sun Y., Cui Z. (2020). The antidepressant-like effects of fluvoxamine in mice involve the mTOR signaling in the hippocampus and prefrontal cortex.Psychiatry Res , 285: 112708. DOI: 10.1016/j.psychres.2019.112708.

14. Palumbo S., Paterson C., Yang F., Hood V.L., Law A.J. (2021). PKBß/AKT2 deficiency impacts brain mTOR signaling, prefrontal cortical physiology, hippocampal plasticity and select murine behaviors. Mol Psychiatry , 26(2): 411-428. DOI: 10.1038/s41380-020-00964-4.

15. Li N., Lee B., Liu R.J., Banasr M., Dwyer J.M., Iwata M., Li X.Y., Aghajanian G., Duman R.S. (2010). mTOR-dependent synapse formation underlies the rapid antidepressant effects of NMDA antagonists.Science , 329(5994): 959-964. DOI: 10.1126/science.1190287.

16. Ma Z., Zang T., Birnbaum S.G., Wang Z., Johnson J.E., Zhang C.L., Parada L.F. (2017). TrkB dependent adult hippocampal progenitor differentiation mediates sustained ketamine antidepressant response. Nat Commun , 8(1): 1668. DOI: 10.1038/s41467-017-01709-8.

17. Liu X.L., Luo L., Mu R.H., Liu B.B., Geng D., Liu Q., Yi L.T. (2015). Fluoxetine regulates mTOR signalling in a region-dependent manner in depression-like mice. Sci Rep , 5: 16024. DOI: 10.1038/srep16024.

18. Haroon E., Miller A.H., Sanacora G. (2017). Inflammation, Glutamate, and Glia: A Trio of Trouble in Mood Disorders.Neuropsychopharmacology , 42(1): 193-215. DOI: 10.1038/npp.2016.199.

19. Trullas R., Skolnick P. (1990). Functional antagonists at the NMDA receptor complex exhibit antidepressant actions. Eur J Pharmacol , 185(1): 1-10. DOI: 10.1016/0014-2999(90)90204-j.

20. Berman R.M., Cappiello A., Anand A., Oren D.A., Heninger G.R., Charney D.S., Krystal J.H. (2000). Antidepressant effects of ketamine in depressed patients. Biol Psychiatry , 47(4): 351-354. DOI: 10.1016/s0006-3223(99)00230-9.

21. Sanacora G., Treccani G., Popoli M. (2012). Towards a glutamate hypothesis of depression: an emerging frontier of neuropsychopharmacology for mood disorders. Neuropharmacology , 62(1): 63-77. DOI: 10.1016/j.neuropharm.2011.07.036.

22. Lener M.S., Niciu M.J., Ballard E.D., Park M., Park L.T., Nugent A.C., Zarate C.A. Jr. (2017). Glutamate and Gamma-Aminobutyric Acid Systems in the Pathophysiology of Major Depression and Antidepressant Response to Ketamine. Biol Psychiatry , 81(10): 886-897. DOI: 10.1016/j.biopsych.2016.05.005.

23. Percie du Sert N., Hurst V., Ahluwalia A., Alam S., Avey M.T., Baker M., Browne W.J., Clark A., Cuthill I.C., Dirnagl U., Emerson M., Garner P., Holgate S.T., Howells D.W., Karp N.A., Lazic S.E., Lidster K., MacCallum C.J., Macleod M., Pearl E.J., Petersen O.H., Rawle F., Reynolds P., Rooney K., Sena E.S., Silberberg S.D., Steckler T., Würbel H. (2020). The ARRIVE guidelines 2.0: Updated guidelines for reporting animal research. Br J Pharmacol, 177(16): 3617-3624. DOI: 10.1111/bph.15193. 
24. Lilley E., Stanford S.C., Kendall D.E., Alexander S.P.H., Cirino G., Docherty J.R., George C.H., Insel P.A., Izzo A.A., Ji Y., Panettieri R.A., Sobey C.G., Stefanska B., Stephens G., Teixeira M., Ahluwalia A. (2020). ARRIVE 2.0 and the British Journal of Pharmacology: Updated guidance for 2020. Br J Pharmacol , 177(16): 3611-3616. DOI: 10.1111/bph.15178.

25. Paxinos G., Franklin K.B.J. (2001). The Mouse Brain in Stereotaxic Coordinates, 2nd ed. California: Academic Press.

26. Alexander S.P.H., Roberts R.E., Broughton B.R.S., Sobey C.G., George C.H., Stanford S.C., Cirino G., Docherty J.R., Giembycz M.A., Hoyer D., Insel P.A., Izzo A.A., Ji Y., MacEwan D.J., Mangum J., Wonnacott S., Ahluwalia A. (2018). Goals and practicalities of immunoblotting and immunohistochemistry: A guide for submission to the British Journal of Pharmacology. Br J Pharmacol , 175(3): 407-411. DOI: 10.1111/bph.14112.

27. Curtis M.J., Alexander S., Cirino G., Docherty J.R., George C.H., Giembycz M.A., Hoyer D., Insel P.A., Izzo A.A., Ji Y., MacEwan D.J., Sobey C.G., Stanford S.C., Teixeira M.M., Wonnacott S., Ahluwalia A. (2018). Experimental design and analysis and their reporting II: updated and simplified guidance for authors and peer reviewers. Br J Pharmacol , 175(7):987-993. DOI: 10.1111/bph.14153.

28. Yamada D., Takeo J., Koppensteiner P., Wada K., Sekiguchi M. (2014). Modulation of fear memory by dietary polyunsaturated fatty acids via cannabinoid receptors. Neuropsychopharmacology, 39(8): 1852-1860. DOI: $10.1038 /$ npp.2014.32.

29. Yamada D., Miyajima M., Ishibashi H., Wada K., Seki K., Sekiguchi M. (2012). Adult-like action potential properties and abundant GABAergic synaptic responses in amygdala neurons from newborn marmosets. $J$ Physiol , 590(22): 5691-5706. DOI: 10.1113/jphysiol.2012.235010.

30. Yamada D., Yanagisawa S., Yoshizawa K., Yanagita S., Oka J., Nagase H. Saitoh A. (2019). Selective agonists of the delta-opioid receptor, KNT-127 and SNC80, act differentially on extinction learning of contextual fear memory in mice. Neuropharmacology , 160: 107792. DOI: 10.1016/j.neuropharm.2019.107792.

31. Gabbott P.L., Warner T.A., Jays P.R., Salway P., Busby S.J. (2005). Prefrontal cortex in the rat: projections to subcortical autonomic, motor, and limbic centers. J Comp Neurol, 492(2): 145-177. DOI: $10.1002 /$ cne.20738.

32. Wallis C.U., Cardinal R.N., Alexander L., Roberts A.C., Clarke H.F. (2017). Opposing roles of primate areas 25 and 32 and their putative rodent homologs in the regulation of negative emotion. Proc Natl Acad Sci U S A , 114(20): E4075-E4084. DOI: 10.1073/pnas.1620115114.

33. Mayberg H.S., Liotti M., Brannan S.K., McGinnis S., Mahurin R.K., Jerabek P.A., Silva J.A., Tekell J.L., Martin C.C., Lancaster J.L., Fox P.T. (1999). Reciprocal limbic-cortical function and negative mood: converging PET findings in depression and normal sadness. Am J Psychiatry, 156(5): 675-682. DOI: 10.1176/ajp.156.5.675.

34. Mayberg H.S., Lozano A.M., Voon V., McNeely H.E., Seminowicz D., Hamani C., Schwalb J.M., Kennedy S.H. (2005). Deep brain stimulation for treatment-resistant depression. Neuron , 45(5): 651-660. DOI: 10.1016/j.neuron.2005.02.014.

35. Drevets W.C., Price J.L., Simpson J.R. Jr., Todd R.D., Reich T., Vannier M., Raichle M.E. (1997). Subgenual prefrontal cortex abnormalities in mood disorders. Nature , 386(6627): 824-827. DOI: 10.1038/386824a0.

36. Fuchikami M., Thomas A., Liu R., Wohleb E.S., Land B.B., DiLeone R.J., Aghajanian G.K., Duman R.S. (2015). Optogenetic stimulation of infralimbic PFC reproduces ketamine's rapid and sustained antidepressant actions. Proc Natl Acad Sci U S A , 112(26): 8106-8111. DOI: 10.1073/pnas.1414728112.

37. Shirayama Y., Hashimoto K. (2017). Effects of a single bilateral infusion of R-ketamine in the rat brain regions of a learned helplessness model of depression. Eur Arch Psychiatry Clin Neurosci , 267(2): 177-182. DOI: $10.1007 / \mathrm{s} 00406-016-0718-1$. 
38. Ghosal S., Hare B., Duman R.S. (2017). Prefrontal Cortex GABAergic Deficits and Circuit Dysfunction in the Pathophysiology and Treatment of Chronic Stress and Depression. Curr Opin Behav Sci, 14: 1-8. DOI: $10.1016 /$ j.cobeha.2016.09.012.

39. Yamada D., Takahashi J., Iio K., Nagase H., Saitoh A. (2021). Modulation of glutamatergic synaptic transmission and neuronal excitability in the prelimbic medial prefrontal cortex via delta-opioid receptors in mice. Biochem Biophys Res Commun , 560: 192-198. DOI: 10.1016/j.bbrc.2021.05.002.

40. Ben-Sahra I., Manning B.D. (2017). mTORC1 signaling and the metabolic control of cell growth. Curr Opin Cell Biol , 45: 72-82. DOI: 10.1016/j.ceb.2017.02.012.

41. Wu D., Yu D., Wang X., Yu B. (2016). F-actin rearrangement is regulated by mTORC2/Akt/Girdin in mouse fertilized eggs. Cell Prolif , 49(6): 740-750. DOI: 10.1111/cpr.12285.

42. McCabe M.P., Cullen E.R., Barrows C.M., Shore A.N., Tooke K.I., Laprade K.A., Stafford J.M., Weston M.C. (2020). Genetic inactivation of mTORC1 or mTORC2 in neurons reveals distinct functions in glutamatergic synaptic transmission. Elife, 9: e51440. DOI: 10.7554/eLife.51440.

43. Younts T.J., Monday H.R., Dudok B., Klein M.E., Jordan B.A., Katona I., Castillo P.E. (2016). Presynaptic Protein Synthesis Is Required for Long-Term Plasticity of GABA Release. Neuron , 92(2): 479-492. DOI: 10.1016/j.neuron.2016.09.040.

44. Sakai Y., Kassai H., Nakayama H., Fukaya M., Maeda T., Nakao K., Hashimoto K., Sakagami H., Kano M., Aiba A. (2019). Hyperactivation of mTORC1 disrupts cellular homeostasis in cerebellar Purkinje cells.Sci Rep , 9(1): 2799. DOI: 10.1038/s41598-019-38730-4.

45. Zeng C., Xing R., Liu J., Xing F. (2016). Role of CSL-dependent and independent Notch signaling pathways in cell apoptosis. Apoptosis , 21(1): 1-12. DOI: 10.1007/s10495-015-1188-z.

46. Sarbassov D.D., Ali S.M., Sengupta S., Sheen J.H., Hsu P.P., Bagley A.F., Markhard A.L., Sabatini D.M. (2006). Prolonged rapamycin treatment inhibits mTORC2 assembly and Akt/PKB. Mol Cell , 22(2): 159-168. DOI: 10.1016/j.molcel.2006.03.029.

47. Fox A.S., Shackman A.J. (2019). The central extended amygdala in fear and anxiety: Closing the gap between mechanistic and neuroimaging research. Neurosci Lett , 693: 58-67. DOI: 10.1016/j.neulet.2017.11.056.

48. Krabbe S., Gründemann J., Lüthi A. (2018). Amygdala Inhibitory Circuits Regulate Associative Fear Conditioning. Biol Psychiatry , 83(10): 800-809. DOI: 10.1016/j.biopsych.2017.10.006.

49. Felix-Ortiz A.C., Burgos-Robles A., Bhagat N.D., Leppla C.A., Tye K.M. (2016). Bidirectional modulation of anxiety-related and social behaviors by amygdala projections to the medial prefrontal cortex.Neuroscience , 321:197-209. DOI: 10.1016/j.neuroscience.2015.07.041.

50. Ko M.J., Chiang T., Mukadam A.A., Mulia G.E., Gutridge A.M., Lin A., Chester J.A., van Rijn R.M. (2021). beta-Arrestin-dependent ERK signaling reduces anxiety-like and conditioned fear-related behaviors in mice. Sci Signal 14(694): eaba0245. DOI: 10.1126/scisignal.aba0245.

Figure Legends

\section{Figure 1.}

KNT-127 exerts antidepressant-like effects through delta opioid receptors and the mTOR signaling pathway in the mice forced swimming test (FST). (A) Dose and time dependent response of KNT-127 (s.c.) for immobility counts in the FST. $\mathrm{n}=12$ for each group. Data are presented as means \pm S.E.M., analyzed using a one-way factorial ANOVA, followed by Dunnett's post-hoc test compared to vehicle groups. ${ }^{*} p<0.01$. (B) Total counts of locomotor activity during $40 \mathrm{~min}$ after the administration of KNT-127 (10 $\mathrm{mg} \mathrm{kg}^{-1}$, s.c.). $\mathrm{n}=9$ for each group. Data are presented as means +- S.E.M., analyzed using Student's $t$-test. n.s.: nonsignificant. (C) Naltrindole $(10 \mathrm{nmol}$, i.c.v. $)$ inhibits the effect of KNT-127 $\left(10 \mathrm{mg} \mathrm{kg}^{-1}\right.$, s.c.) in the FST. $\mathrm{n}=10,9,9,9$ each from the left. Each numerical difference from 10 was discarded from 
the data. (D) Rapamycin (0.2 nmol, i.c.v.) abolishes the effect of KNT-127 (10 $\mathrm{mg} \mathrm{kg}^{-1}$, s.c.) in the FST. $\mathrm{n}=9,10,8,8$ each from the left. Each numerical difference from 10 was discarded from the data. Data are presented as means +- S.E.M., analyzed using a two-way factorial ANOVA, followed by Dunnett's post-hoc test compared to vehicle groups. ${ }^{*} p<0.01$.

Figure 2.

KNT-127 activates the mTOR signaling related proteins in specific brain regions . (A-C) Integrated optical density (IOD) of protein bands in the Western blotting, normalized to the "vehicle + vehicle"-treated group. p-Akt and total Akt (A), p-ERK and total ERK (B), and p-p70S6K and total p70S6K (C). $\mathrm{n}=5$ for each group. Each representative image of protein bands was from the same blot. Data are presented as means +- S.E.M., analyzed using two-way factorial ANOVA, followed by Dunnett's post-hoc test compared to vehicle groups. ${ }^{*} p<0.01$.

\section{Figure 3.}

Antidepressant-like effects of KNT-127 are involved in the PI3K pathway. (A) A PI3K inhibitor, LY294002, (20 nmol, i.c.v.) blocked the effect of KNT-127 (10 mg kg-1, s.c.) in the forced swimming test (FST). $\mathrm{n}=10,9,9,9$ each from the left. Each numerical difference from 10 was discarded from the data. (B) A MEK inhibitor, U0126, (20 nmol, i.c.v.) did not affect the effect of KNT-127 (10 $\mathrm{mg} \mathrm{kg}^{-1}$, s.c.) in the FST. $\mathrm{n}=10,9,10,9$ each from the left. Each numerical difference from 10 was discarded from the data. Data are presented as means +- S.E.M., analyzed using two-way factorial ANOVA, followed by Dunnett's post-hoc test compared to vehicle groups. ${ }^{*} p<0.01$.

\section{Figure 4.}

KNT-127 directly produces antidepressant-like effects in the infralimbic cortex (IL-PFC) in the forced swimming test (FST). (A, B) Local infusion of KNT-127 (1 mM, $0.2 \mu \mathrm{L} /$ side) in the prelimbic cortex (A) or IL-PFC (B). Left panel: points of cannulas, images were modified from the mouse brain atlas by Paxinos and Franklin (25); right panel: immobility counts in the FST. $\mathrm{n}=8$ for each group. Two subjects for each group were discarded from the data. Data are presented as means \pm S.E.M., analyzed using the Student's $t$-test. ${ }^{*} p<0.01$.

Figure 5.

KNT-127 accelerates glutamate release in the infralimbic cortex with involvement of the mTOR signaling pathway. Each dataset expressed vehicle pretreated group (A-E) and rapamycin pretreated group (F-J). (A, F) Representative trace of miniature excitatory postsynaptic currents. (B, G) Mean frequency. (C, H) Mean amplitude. (D, I) Mean rise time. (F, J) Mean decay time. vehicle, $\mathrm{n}=12$ cells from eight mice; rapamycin, $\mathrm{n}=10$ cells from six mice. Data are presented as means \pm S.E.M., analyzed using paired sample $t$-test. ${ }^{*} p<0.01$.

\section{Figure 6.}

KNT-127 suppressed GABA release in the infralimbic cortex with involvement of the mTOR signaling pathway. Each dataset expressed vehicle pretreated group (A-E) and rapamycin pretreated group $(\mathrm{F}-\mathrm{J})$. (A, F) Representative trace of miniature inhibitory postsynaptic currents. (B, G) Mean frequency. (C, H) Mean amplitude. (D, I) Mean rise time. (F, J) Mean decay time. vehicle, $\mathrm{n}=12$ cells from seven mice; rapamycin, $\mathrm{n}=11$ cells from six mice. Data are presented as means \pm S.E.M., analyzed using the paired sample $t$-test. ${ }^{*} p<0.01$.

\section{Figure 7.}

Schematic diagram of a hypothesized functional mechanism for the antidepressant-like effects of the selective delta opioid receptor (DOP) agonist, KNT-127. (A) Activation of DOPs attenuates GABA release through the PI3K-Akt-mTOR-p70S6K signaling pathway in the infralimbic cortex. Consequently, glutamate release accelerated and evoked antidepressant-like actions. 
Figure 1
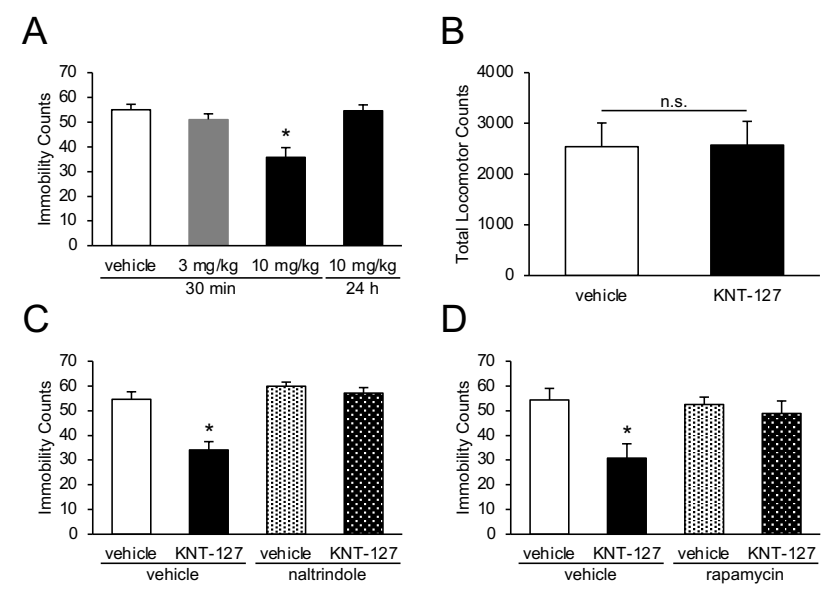
Figure 2
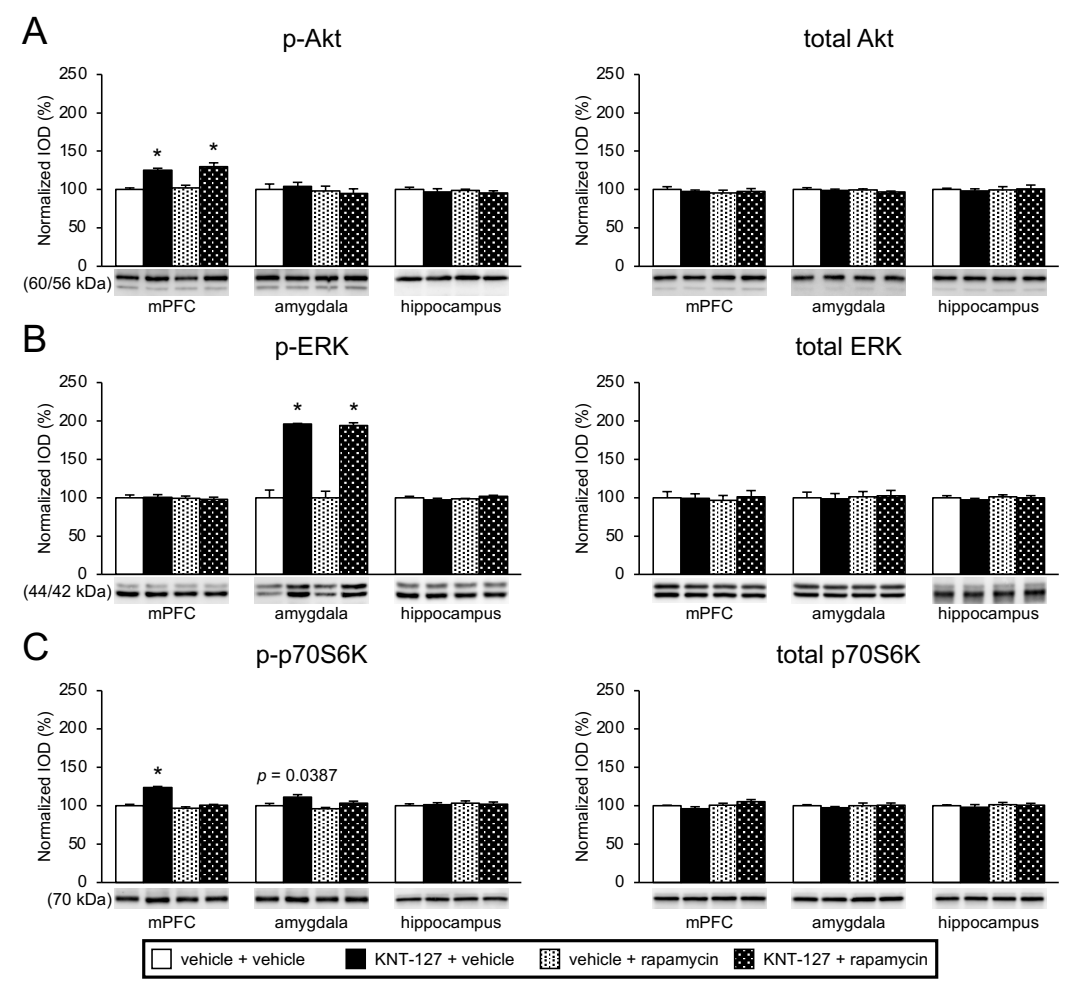
Figure 3

A

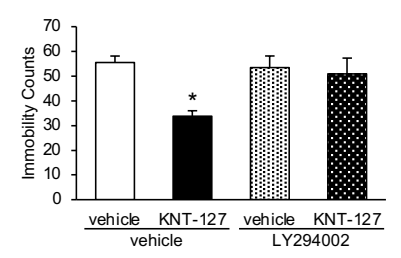

B

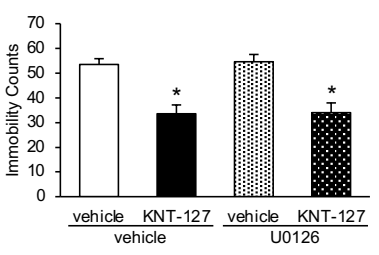


Figure 4




Figure 5

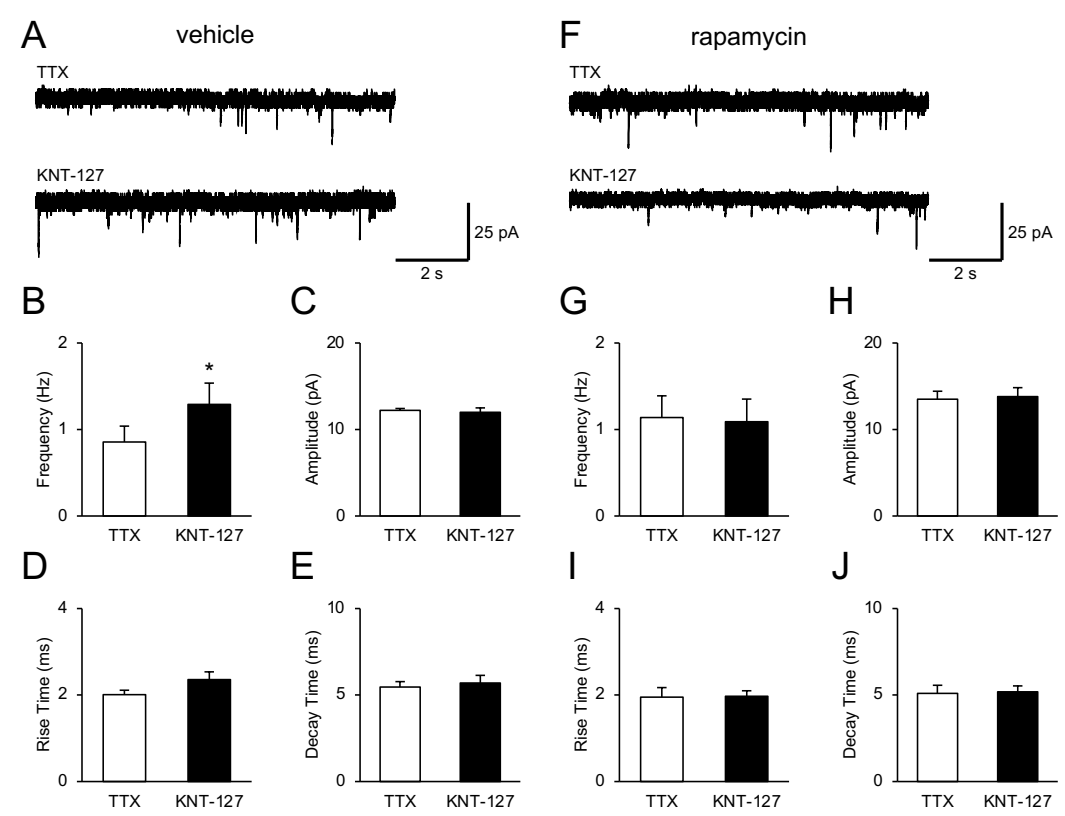


Figure 6
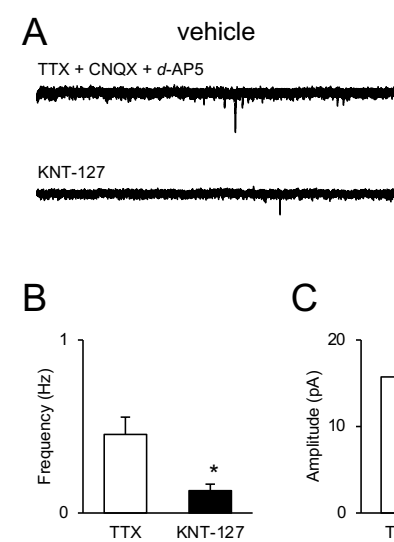

D

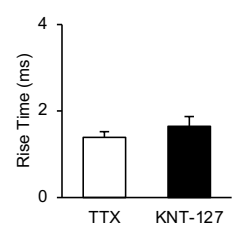

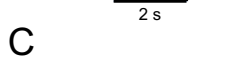

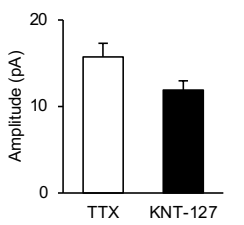

E

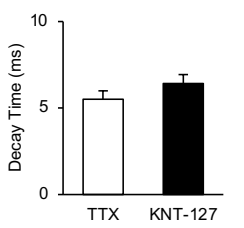

F

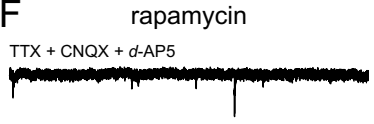

KNT-127

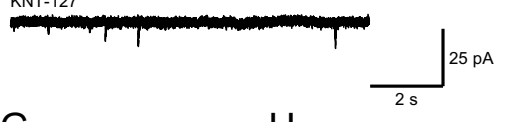

G

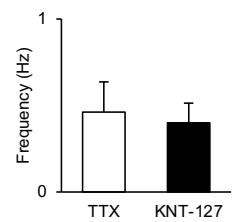

I

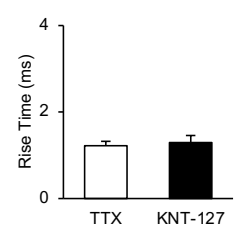

$\mathrm{H}$
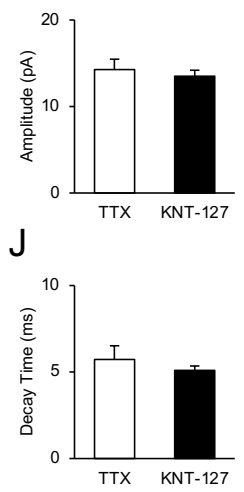
Figure 7

A

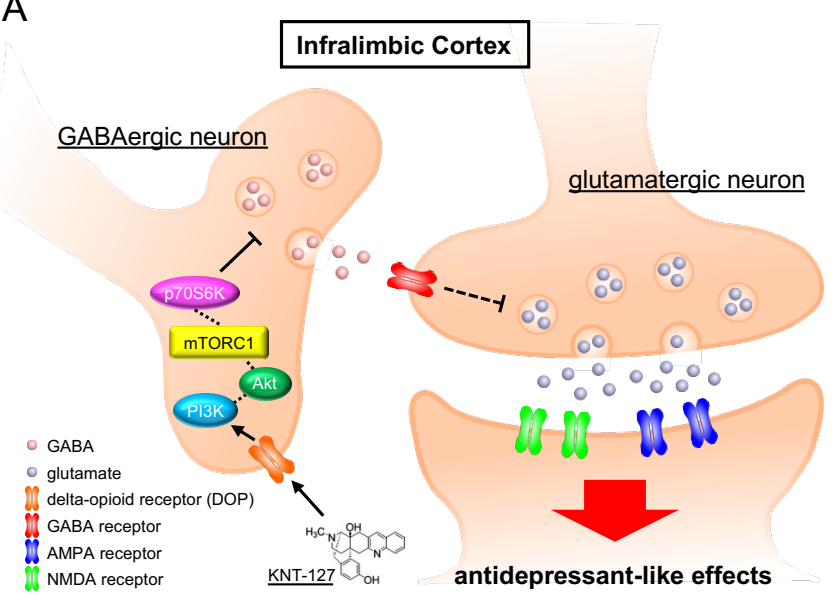

